\title{
Kernos
}

Revue internationale et pluridisciplinaire de religion grecque antique

5 | 1992

Varia

\section{Artémis de Lousoi : les fouilles autrichiennes}

\section{Veronika Mitsopoulos-Leon}

URL : http://journals.openedition.org/kernos/1051

DOI : 10.4000/kernos.1051

ISSN : 2034-7871

\section{Éditeur}

Centre international d'étude de la religion grecque antique

Édition imprimée

Date de publication : 1 janvier 1992

ISSN : 0776-3824

\section{Référence électronique}

Veronika Mitsopoulos-Leon, «Artémis de Lousoi : les fouilles autrichiennes », Kernos [En ligne],

5 | 1992, mis en ligne le 19 avril 2011, consulté le 01 mai 2019. URL : http://journals.openedition.org/ kernos/1051; DOl : 10.4000/kernos.1051 


\section{ARTÉMIS DE LOUSOI : LES FOUILLES AUTRICHIENNES}

Dans le cadre d'un colloque sur la religion grecque antique et plus spécialement sur les cultes orgiastiques, il s'impose de s'interroger encore une fois sur le culte de l'Artémis Hémera de Lousoi. Ainsi seront présentés quelques résultats récents des fouilles autrichiennes et quelques-uns des petits objets qui pourraient éventuellement nous donner une information complémentaire sur l'aspect de la déesse et son culte local.

En 1898 et 1899, A. Wilhelm et W. Reichel dirigeaient trois campagnes de fouilles dans le sanctuaire de la déesse ${ }^{1}$. Depuis 1981, c'est le même institut qui continue la recherche à Lousoi, dans le temple et aussi dans une partie de la ville ${ }^{2}$. Les sources sont connues et ont été bien discutées ${ }^{3}$; aussi me limiterai-je à quelques détails qui me semblent avoir de l'importance pour les explications qui suivront.

$\mathrm{Au}$ Ve siècle, Bacchylide, qui probablement connaissait bien la région, dans son épinikion pour un certain Alexidamos de Métaponte, vainqueur aux Pythies ${ }^{4}$, nous donne quelques précieuses informations : il décrit l'épisode de la guérison des filles du roi Proitos de Tirynthe frappées - par Héra ou par Dionysos - de folie. Ainsi, le père désespéré, après un bain sacral dans le fleuve de Lousos, prie la déesse d'intervenir auprès d'Héra.

1 W. Reichel - A. Wilhelm, Das Heiligtum der Artemis zu Lousoi, in ÖJh, 4 (1901), p. 1-89.

2 F. Glaser, Lousoi, in ÖJh, 53 (1981/1982), Grabungen, p. 24; V. Mitsopoulos-Leon, Lousoi, Ergebnisse neuer Forschungen, in ÖJh, 56 (1985), p. 93-98; V. MITSOPOULOS-LEON - F. GLASER, in ÖJh, 57 (1986/1987), Grabungen, p. 18-22. Les mêmes, in ÖJh, 58 (1988), Grabungen, p. 14-18; V. Mitsopoulos-LeON, in ÖJh, 59 (1989), Grabungen, p. 11-14. La même, in $\ddot{O} J h, 60$ (1990), Grabungen, p. 32-36. Cf. aussi in $B C H$, rapports des fouilles depuis $1983, B C H, 108$ (1984), p. 771-772, et $A R$ (1986-1987), p. 20 (pour 1986); $A R$ (1987-1988), p. 24 (pour 1987); $A R$ (1989-1990), p. 20 (pour 1989).

REICHEL et WILHELM, loc. cit., p. 3-7; M. JosT, Sanctuaires et cultes d'Arcadie, Paris, 1985 (Et. Pélop., 9), p. 46-51, 420-425; R. STIGLITZ, Die großen Göttinen Arkadiens; Der Kultname und seine Grundlagen, in Sonderschriften hrsg. vom österr. Archäologischen Institut, XV, 1967; R. GINOUVES, Balaneutikè, 1962, p. 327, n. 6; U. SiNN, Ein Fundkomplex aus dem Artemis-Heiligtum von Lousoi im Badischen Landesmuseum?, in Jb Staatl. Kunstsammlungen Bawü, 17 (1980), p. 25-40.

4 BACCHYL., Epin., X (XI), 92-112. 
La guérison achevée, les filles reconnaissantes consacrent un téménos et un autel, sur lequel elles sacrifient de petits animaux, brebis et chèvres; ensuite elles organisent des chœurs de femmes, une institution habituelle dans le culte d'Artémis dans le Péloponnèse ${ }^{5}$. C'est ainsi que Bacchylide dessine l'image d'un sanctuaire très ancien, où dominent le téménos et l'autel avant que le temple n'occupe la place dominante ${ }^{6}$.

Ensuite et pour lier Lousoi à Métaponte, patrie d'Alexidamos, Bacchylide nous parle du départ des Achéens pour Métaponte, leur nouvelle patrie. Avant de quitter la Grèce, ils se seraient rassemblés dans le sanctuaire de l'Artémis qu'ils priaient de les accompagner dans leur nouvelle patrie ${ }^{7}$, événement à situer au VIIe siècle, époque de la colonisation ${ }^{8}$. Ce récit touchant montre déjà l'importance relative du petit sanctuaire et atteste que Lousoi, à cette époque, appartenait probablement à la région d'Achaïe.

Callimaque mentionne un temple ${ }^{9}$. Polybe ${ }^{10}$, entre autres, nous informe que la ville avait le droit d'asile, non respecté par les Étoliens; ceux-ci emportaient aussi les troupeaux d'animaux qui se trouvaient sous la protection de la déesse. En l'honneur de la déesse se tenaient des jeux, les Hémérasia, dont parlent encore les inscriptions du Ier siècle ap. J.-C. ${ }^{11}$ Dans une inscription de Thuria, le stade et le diaulos sont spécialement mentionnés ${ }^{12}$.

Et de la part de Pausanias? Peu de nouvelles : "Il n'y a même plus de ruines à voir de la ville» de son temps ${ }^{13}$. Quant à la guérison des filles folles, il confond la tradition qui attribue cette action à Mélampous et qui est à situer ailleurs, entre autres dans la ville voisine de Kleitor ${ }^{14}$.

5 Pour les chœurs de femmes cf. Jost, op. cit., p. 421, n. 2; B. Schweitzer, Die geometrische Kunst Griechenlands, 1969, p. 165 : pour Artémis Orthia, Karyatis, Limnatis, Kordax (Élis), Alphaia; C. CALAME, Les chæurs de jeunes filles en Grèce archaïque, Rome, 1977, p. 216 sq.

6 SchweItzer, op. cit., p. 233.

7 BACCHYL., Epin., X (XI), 113-117.

8 SinN., loc. cit., p. 36, n. 69.

9 Callim., Hymne à Artémis, v. 233-236.

10 Polybe, IV, 18, 9; 19, 4; IV, 25, 4; IX, 34, 9.

11 Cf. JosT, op. cit., p. 47, 48 n. 1; Mitsopoulos-LEON, in ÖJh, 56 (1985), p. 95, n. 29. Pour le Ier s. ap. J.-C. cf. SEG, XVII (1960), nº 628.

12 I.G., V 1, 1387 de Thuria en Messénie. L. RoBert, in RPhil, 56 (1930), p. 35 36.

13 PAus., VIII, 18, 7-8.

14 JosT, op. cit., p. 421 sq. 
Rendons-nous sur place : dans la région montagneuse du nord de l'Arcadie s'étend aux pieds du Chelmos la haute vallée de Soudena, entourée d'une chaîne de montagnes. Sur la pente du prophète Ilias s'étalait la ville de Lousoi et son sanctuaire ${ }^{15}$ d'Artémis Héméra, "la calmante» 16. Vers le nord, la ville ancienne de Kynaitha, aujourd'hui Kalavryta et vers le sud, Kleitor.

À un niveau inférieur, les premiers autrichiens dégageaient les restes de quelques bâtiments ${ }^{17}$ : d'une fontaine qu'on désigne aussi comme bassin pour le culte ${ }^{18}$; puis d'un propylon et d'un bâtiment généralement appelé bouleutérion ou exèdre (pour des statues) ${ }^{19}$.

$\mathrm{Au}$ sommet de la colline qui monte derrière ces bâtiments, en position dominante, s'élevait le temple de la déesse ${ }^{20}$. Reichel et Wilhelm avaient déjà dégagé la plupart des fondations du temple, dressé le plan ${ }^{21}$ et proposé une reconstitution ${ }^{22}$. Notre Institut en poursuivit la fouille après nettoyage ce qui nous permet de présenter un nouveau plan, avec quelques corrections ${ }^{23}$. En bref : le temple présente une forme hors du commun : une nef centrale flanquée de deux ailes le long des murs longs. À l'extérieur étaient appliqués des piliers tandis qu'à l'intérieur se trouvaient des demi-colonnes prolongées. Une porte latérale donnait accès de la nef sud au naos 24 . Nos recherches récentes ont démontré que l'existence d'une deuxième porte en vis-à-vis, du côté nord, telle qu'elle fut proposée dans la reconstitution de la première fouille, est à exclure définitivement ${ }^{25}$.

Reichel et Wilhelm placent quatre colonnes à l'entrée de même que quatre colonnes du côté ouest. Ils divisent la nef centrale en naos, pronaos

15 G. Papandreou, Azanias, 1886, p. 77-78; Reichel-Wilhelm, loc. cit., p. 1 sq.

16 Sur l'interprétation du nom cf. STIGLITZ, op. cit., p. 103-105, par contre JosT, op. cit., p. 420, traduit la «Douce» et y voit une «désignation propitiatoire par antiphrase».

17 REICHEL-WILHELM, loc. cit., p. 15-24.

18 Ginouves, op. cit., p. 383; K. Dowden, Death and the Maiden. Girls' Initiation Rites in Greek Mythology, 1989, p. 91.

19 Jost, op. cit., p. 48 et n. 6; H. LAUTER, Die Architektur des Hellenismus, 1986, p. 210.

20 Reichel-WILHELM, loc. cit., p. 24-33; Jost, op. cit., p. 49.

21 ReICHEL-WILHELM, loc. cit., p. 26-27, fig. 16.

22 Plan restitué : REICHEL-WILHELM, loc. cit., p. 24, fig. 14.

23 Cf. note 1.

24 REICHEL-WILHELM, loc. cit., p. 30, fig. 14.

25 Mitsopoulos-LEON, Grabungen 1989, in ÖJh, 60 (1990), Grabungen, p. 32-36. 
et opisthodome $e^{26}$. C'est ici que nous corrigeons encore une fois ${ }^{27}:$ au milieu du mur qui sépare le naos de la chambre arrière, manquent quelques blocs. Ces blocs-ci n'ont jamais été prévus, jamais été posés, donc il faut probablement reconstituer à cette place un linteau, donc une porte : une porte conduisant à un adyton. Le temple de l'Artémis de Lousoi serait donc muni d'une niche sacro-sainte, accueillant le trésor ou des personnes d'exception, par exemple le prêtre ou un myste, et appartenant ainsi à une série de temples avec adyton comme ceux de l'Artémis de Brauron, à Aulis, de la Tauropolos, de Kombothekra près d'Olympie ou même de l'Aphaia à Égine 28 . Reichel et Wilhelm datent toutes les fondations de la fin du IVe ou du IIIe s. ${ }^{29}$ Quelques fragments plus anciens leur laissaient supposer l'existence d'un temple précurseur, peut-être du VIe s., à chercher dans les fondations du temple hellénistique ${ }^{30}$. Nous n'avons pas $\mathrm{pu}$ trouver les vestiges d'un bâtiment précurseur, dont cependant l'existence n'est pas douteuse. Peut-être de grands travaux d'aplanissement, des mouvements de terre précédaient-ils la construction du nouveau temple? Une date pour la construction de l'aile sud nous est donnée par des objets trouvés dans la fosse de construction ${ }^{31}$ : entre autres une monnaie et un fragment de lampe de la moitié du IIIe s. et c'est un terminus ante quem non au moins pour l'aile sud.

Quand nous examinons les petits objets d'un sanctuaire, nous devons tenir compte de quelques faits: il faut observer la différence entre les offrandes qu'on trouve dans plusieurs sanctuaires et qui sont offertes aussi à d'autres dieux (par ex. les statuettes de bœufs, de chevaux, de différents oiseaux, certains bijoux) et ces objets qui sont caractéristiques d'un sanctuaire particulier ${ }^{32}$. Il faut aussi discerner entre des objets

REICHEL-WILHELM, loc. cit., fig. 14.

Mitsopoulos-Leon, Grabungen 1989.

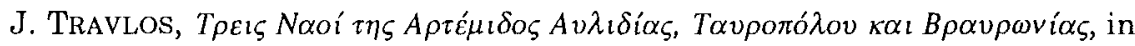
NFGH (1976), p. 197 sq.; U. SinN, Das Heiligtum der Artemis Limnatis bei Kombothekra, in $A M, 93$ (1978), p. 48 sq.; E.-L. SCHWANDNER, Der älteste Porostempel der Aphaia auf Ägina, 1985, p. 108 sq. Contre Travlos, cf. M. Hollinshead, Against Iphigeneia's Adyton in Three Mainland Temples, in AJA, 89 (1985), p. 419 sq.

29 REICHEL-WILHELM, loc, cit., p. 20, 32.

30 REICHEL-WILHELM, loc. cit., p. 32.

31 Mitsopoulos-Leon - Glaser, Grabungen 1987, in ÖIh, 58 (1988), p. 14 sq., fig. 4.

32 Pour les objets des sanctuaires en Arcadie, cf. M. VoYatzi, The Early Sanctuary of Athena Alea at Tegea and other Archaic Sanctuaries in Arcadia, Göteborg, 1990 (SIMA Pocket-book, 97), passim. 
singuliers qui furent apportés par des visiteurs de leur patrie et ceux qui furent fabriqués par des artisans immigrés ou par les habitants de la région, peut-être même à proximité du temple et que les fidèles pouvaient acheter sur place.

La situation des petits objets de Lousoi est un peu compliquée : ceux de la vieille fouille autrichienne se trouvent au Musée national d'Athènes $^{33}$. Les objets de la fouille courante sont dans le dépôt local ${ }^{34}$. Enfin, un grand groupe d'objets proviennent des fouilles clandestines et sont dispersés dans d'innombrables musées et collections à l'étranger ${ }^{35}$. Trois statuettes provenant des fouilles clandestines et vendues à l'étranger représentent les dieux présents à Lousoi comme généralement dans toute l'Arcadie.

La statuette de bronze du Liebighaus à Frankfurt a été assez discutée ${ }^{36}$. Avec sa forme de pilier; avec sa petite cape qui ressemble à celle des bergers de l'Arcadie, mais aussi à l'épibléma dédalique que portent les sphyrelata de Dréros en Crète ${ }^{37}$; avec sa coiffure de page, coupée toute droite sur le front, elle rappelle les statues de style subgéométrique ou dédalique. Mais des éléments plus récents, comme son profil plutôt classique et l'indication, même maladroite, d'une pondération, la datent au plus tôt vers le commencement du Ve s. Ainsi elle serait une création de l'époque du haut classicisme et refléterait l'œuvre dédalique qu'était la statue de culte de la déesse ${ }^{38}$.

Qu'il y ait eu sûrement une tradition locale est attesté par les petites statuettes primitives en terre cuite ${ }^{39}$ qui portent la coiffure caractéristique de page de la statuette de bronze et soulignent ainsi une différence éclatante avec les autres statuettes de cette sorte qu'on trouve ailleurs.

33 Reichel-Wilhelm, loc. cit, p. 91, 133, 143, 155, 242, et passim.

34 Cf. les rapports de fouille (note 2 ).

35 P. ex. Sinn, loc. cit., passim (note 3), qui publie des exemples du complexe à Karlsruhe.

36 A. FURTWÄNGLER, Arkadische Bronzestatuetten, in Neue Denkmaler antiker Kunst 2, SB München, (1899) 2, p. 571 sq. (= Kleine Schriften von A. Furtwängler, 2 [1913], 462 sq.); R. Tölle-Kastenbein, Frühklassische Peplosfiguren, Originale, 1980, p. 149-152; LIMC, II, 1 (1984), 633, v. Artémis (L. KAHIL); P. BoL, Die "Artemis von Lousoi", eine klassische Wiedergabe eines frühgriechischen Kultbildes, in Kanon, Festschrift E. Berger, AK (1988), p. 76 80.

37 C. Davaras, Die Statue aus Astritsi, in AK, 8. Beiheft (1972), p. 63; Bol, loc. cit., p. 76.

38 JosT, op. cit., p. 50 et 421, préfêre y voir des dédicantes.

39 REICHEL-WILHELM, loc. cit., fig. 26-29, 31. 
Le frère d'Artémis était aussi présent : l'Apollon Béarn ${ }^{40}$ porte l'arc; la coiffure ressemble un peu à celle de sa sœur. Quant à l'inscription sur le dos et la jambe gauche, elle pose des problèmes ${ }^{41}$.

Dans tous les sancutaires arcadiens se trouve le dieu Pan qui surveille les troupeaux de moutons et de chèvres. Un des plus beaux exemples de l'art péléponnésien du Ve, IVe s. se trouve maintenant à Berlin ${ }^{42}$.

Parmi les objets les plus nombreux à Lousoi nous comptons les masques et les protomés ${ }^{43}$. Quelques exemplaires de ce type de visage oval, les lèvres pleines et les yeux entourés de paupières lourdes, appartiennent au groupe nord-péloponnésien ${ }^{44}$, daté fin VIe/commencement Ve s. De l'autre côté, un fragment est pour le moment unique à Lousoi ${ }^{45}$ : son bord supérieur bien travaillé ressemble à l'embouchure d'un vase. Avec ses boucles longues et plates sur le front et les tempes, il est proche du kouros de Tenéa ${ }^{46}$ ou d'un sphinx de la région de Corinthe, probablement de la main du même maître et daté au troisième quart du VIe s. ${ }^{47} \mathrm{Il} \mathrm{y} \mathrm{a}$ aussi des parallèles en Italie du Sud ${ }^{48}$. Deux bras tendus avec main ouverte ou poing fermé appartenaient à des statues de hauteur moyenne.

40 FURTWÄNGLER, loc. cit., p. 566-571; la statuette fut vendue : cf. Antiquités et Objets d'Art Coll. de Martine, Comtesse de Béhague, Prov. de la succession du Marquis de Ganay, Monaco, 5 déc. 1987, n 120, p. 96-101.

41 Cf. note 40. L. DuboIs, Recherches sur le dialecte arcadien, Louvain-la-Neuve, 1986 (BCILL, 2), p. 214; HILLER, IG, V, 403; L.H. JEFFERY, The Local Scripts of Archaic Greece, $1990^{2}$, p. 210-211, n $1 ; 215, n^{\circ} 14$.

42 Berlin, Musée de Charlottenburg, 8264; K.A. Neugebauer, Kat. Berlin, Bronzen, 2, 1951, $\mathrm{n}^{\circ} 16$ et pl. 16; U. Gehrig, A. GrEIFENHAGEN, N. Kunisch, Führer durch die Antikenabteilg., 1968, p. 152, pl. 20; JosT, op. cit., p. 465.

REICHEL-WILHELM, loc. cit., 41 et fig. 39-45.

44 F. CROISSANT, Les protomés féminines archaïques, recherches sur les représentations du visage dans la plastique grecque de 550 à 480 av. J.-C., Paris, 1983 (BEFAR, 250), p. 359-370.

45 TK 18/87, à l'est du temple 2 frms.; le bord supérieur bien travaillé, comme l'embouchure d'un vase; argile rosée, grise au centre; fine, quelques petites inclusions blanches; $h=0,055 \mathrm{~m}$. Cf. un exemplaire de Métaponte, W. SchÜrmANN, Kat. ant. Terrakotten im Badischen Landesmuseum Karlsruhe, Göteborg, 1989 (SIMA, 84), p. 92, nº 313.

46 G.M.A. Richter, Kouroi, Archaic Greek Youths, 1960, p. 84-85, fig. 245-246; CROISSANT, op. cit., p. 246.

47 E. Protonotariou-Deilaki, in $A A, 6$ (1973), p. 181 sq., fig. 1, 2. ArchRepts, 1979-1980, p. 24, photo sur couverture.

48 SchürmanN, op. cit., n 313 (Métaponte); M. BARRA Bagnasco, Protomi in Terracotta da Locri Epizefiri, 1986, p. 89-91, n 141. 
Nous ajoutons ici la belle tête d'un Apollon, fin VIe/commencement Ve s., haute de $9 \mathrm{~cm}$ et appartenant à une statuette d'à peu près 60 à $70 \mathrm{~cm}$. On trouve un exemple comparable à Métaponte ${ }^{49}$. Les femmes debout, fleur ou petit daim dans les mains et les bras, types de Corinthe, sont aussi représentées à Lousoi ${ }^{50}$.

Parmi les riches trouvailles en métal, je mentionne avant tout quelques objets qui pourraient facilement avoir été fabriqués sur place : par ex. des coqs ou des mains, en feuille de bronze ${ }^{51}$; le décor simple fut empressé sans grande difficulté dans la surface. Du IIIe s. datent de petits coffres en bronze ${ }^{52}$; on en a trouvé de pareils à Dodone, à Samothrace, en Sicile. Deux fragments de Lousoi portent une inscription ${ }^{53}$; c'est important de mentionner que les noms des donateurs, là où ils sont conservés, sont toujours masculins. Dans l'un des cas, c'est un Arexidamos qui offre la petite boîte : il rappelle le vainqueur aux Pythies du poème de Bacchylide ${ }^{54}$. La présence dans le temple et dans le culte de garçons ou de jeunes hommes semble ainsi assurée ${ }^{55}$. Il me semble remarquable (qu'à part deux fragments) il n'y ait pas de lampes dans le temple ${ }^{56}$.

De l'autre côté, on compte des fragments de vases, par ex. des lecanides, des pyxides, des oinochoes, des vases à boire, etc. Les plus

49 Mitsopoulos-LeON - Glaser, Grabungen 1986, in ÖJh, 57 (1986/1987), p. 21, fig. 5 (avant nettoyage complet); pour l'exemple de Métaponte, cf. R.A. HigaINS, Greek Terracottas, 1967, p. 55, pl. 22 d.

50 Cf. A. STILlwell, Corinth, XV 2 (1952), p. 86 sq., 88.

51 ReICHEL-WILHELM, loc. cit., p. 61 et 62,65 et 66 ; VoYATZI, op. cit., pl. 171 (Lousoi), pl. 172 (Asea); p. 264, elle accepte aussi un atelier dans le voisinage du temple, "perhaps the second largest known center in Arcadia in geometric times".

52 Mitsopoulos-LeON - Glaser, Grabungen 1987, in ÖJh, 58 (1988), p. 14, fig. 3a, b; V. Mitsopoulos-LeOn, Kästchen für Artemis, in EYMOYSIA, Ceramics and Iconographic Studies in Honour of A. Cambitoglou, MedArch Suppl., 1 (1990), p. 137-140.

53 MitSOPOULOS-LEON, in EYMOYEIA, p. 137. REICHEL-WILHELM mentionnent loc. cit., 70, $\mathrm{n}^{\circ} 7$, le nom 'A $\lambda \varepsilon \xi_{1}$... dans une inscription.

54 BACCHYL., Epin., X (XI).

55 Cf. U. SinN, Aphaia un die "Aegineten", zur Rolle des AphaiaHeiligtums im religiösen und gesellschaftlichen Leben der Insel Ägina, in MDAI(A), 102 (1987), p. 130-167, 138 sq., fig. 3 (banderoles de Lousoi); Sinn est de l'opinion que ces banderoles en feuille de bronze étaient destinées à serrer les boucles des jeunes gens, dédiées pendant les rites d'initiation.

56 Deux exemplaires en bronze, cf. REICHEL-WILHELM, loc. cit., fig. 120, 121; pour le fragment en argile mentionné ici, cf. n. 34. 
nombreux sont des vases miniatures ${ }^{57}$ et des modèles stéréotypés de pyxides ${ }^{58}$ avec ses anses verticales et couvercle pointu fixé sur le corps du vase. Nous connaissons la forme - la stamnos-pyxis - depuis l'époque géométrique $^{59}$. Peut-être ce type de vase jouait-il un certain rôle dans le culte ou était-il une offrande de préférence pour la déesse de Lousoi.

Pour finir je présente un fragment d'une amphore, conservé en très mauvais état : la couleur noire a presque entièrement disparu, les incisions sont très négligées, le décor difficile à reconnaître ${ }^{60}$. Sur ce fragment on discerne un pied avec le sabot d'un animal, marchant vers un perirrhanterion avec bord décoré en zig-zag. Derrière lui s'élève un objet, oblong sur une base; qu'il s'agisse de la représentation d'un objet architectural ou statuaire, il semble étroitement lié au perirrhanterion.

L'eau, nous le savons, jouait un rôle important dans le culte de la déesse Artémis. À l'entrée des temples se trouvaient des perirrhanteria «jouant un peu le même rôle que les bénitiers des églises chrétiennes» 61 ou "to mark special transitions between secular and sacred territory" 62 . La tradition de Lousoi même ${ }^{63}$ évoque l'importance de l'eau : le roi Proitos prend son bain de purification dans le beau fleuve Lousos. De petites statuettes d'hydrophores, jeunes filles portant une hydrie sur la tête ${ }^{64}$, ont été trouvés dans la fouille. Si mon interprétation du fragment d'amphore est acceptée, nous aurions ici la représentation d'une cérémonie de purification à situer peut-être à la proximité du temple luimême.

57 REICHEL-WILHELM, loc. cit., p. 44.

58 ReICHEL-WILHELM, loc. cit., p. 44 sq.

59 J.H. Coldstream, Greek Geometric Pottery, p. 168, passim. B. Bohen, Kerameikos, XIII : Die geometrischen Pyxiden, 1988, p. 5 sq.,13.


restauration non encore complétée; argile beige, fine; décor: incision et noir; dessin par Cl. Lepeniotis; le vase est proche d'un groupe de Corinthe, cf. I. JuCKer, Frauenfest in Korinth, in $A K, 6$ (1963), p. 47-61. Ch. PAPADOPOUlou-

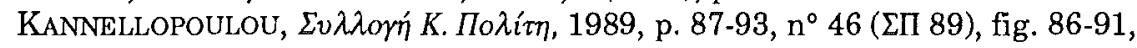
pl. 14.

61 Ginouves, Balaneutikè, 1962, p. 299 sq. et passim.

62 S. Guettel-Cole, The Uses of Water in Greek Sanctuaries, in R. HÄGG, N. Marinatos, G. Nordquist (eds), The Early Cult Practice. Proceedings of the 5th. Intern Symposium, Göteborg, 1988 (Skrifter utgivna av Svenska Institutet i Athen, $4^{\circ}, 38$ ), p. 161-165.

63 Pour l'eau dans le culte d'Artémis à Lousoi et le problème de la source dans la légende de Mélampous, cf. JosT, op. cit., p. 420-425.

64

REICHEL-WILHELM, loc. cit., fig. 51. 
Dressons un bilan pour l'Artémis de Lousoi : elle est potnia thêrôn, elle protège les animaux; elle intervient auprès d'Héra pour que les filles du roi Proitos soient guéries - elle est la Hémera, celle qui est calmante. Dans une autre tradition, là où Mélampous est guérisseur des filles, c'est quand même dans le temple de l'Artémis qu'elles se rassemblent pour la remercier ${ }^{65}$. Et de quelle maladie s'agit-il ? C'était la mania, envoyée ou par Héra ou par Dionysos. Par Héra, parce qu'elles s'étaient vantées devant la déesse. Par Dionysos, parce qu'elles refusaient d'être initiées à son culte. Aussi étaient-elles condamnées à errer dans les montagnes de l'Arcadie. Leurs souffrances prirent fin grâce à l'intervention de l'Artémis Hémera de Lousoi, dans son sanctuaire ${ }^{66}$; cette force guérisseuse et calmante est aussi indiquée par la fleur de pavot que tenait à la main la statuette trouvée à Kalavryta et maintenant conservée à Berlin ${ }^{67}$.

Österreichisches Archäologisches Institut

Veronika MITSOPOULOS-LEON

Leoforos Alexandras, 26

GR - 10683 ATHEN

65 Cf. supra n. 4.

66 Cf. Jost, op. cit., p. 424-425, Artémis soulage les maladies.

67 FurTwäNGLER, loc. cit., p. 575 sq.; maintenant à Berlin, Staatl. Museen, 7644; K. Neugebauer, op. cit., p. 22 sq., n 13, pl. 14; Gehrig, Greifenhagen, KunISCH, op. cit., p. 133; RE, XV 2 (1932), c. 2433-2446, v. Mohn (Steier), la déesse porte le pavot en sa qualité de guérisseuse des maladies psychiques et nerveuses; cf. aussi JOST, op. cit., p. 403; la tête de femme en terre cuite de Métaponte déjà mentionnée (cf. supra n. 45) porte une couronne de fleurs de pavot. 


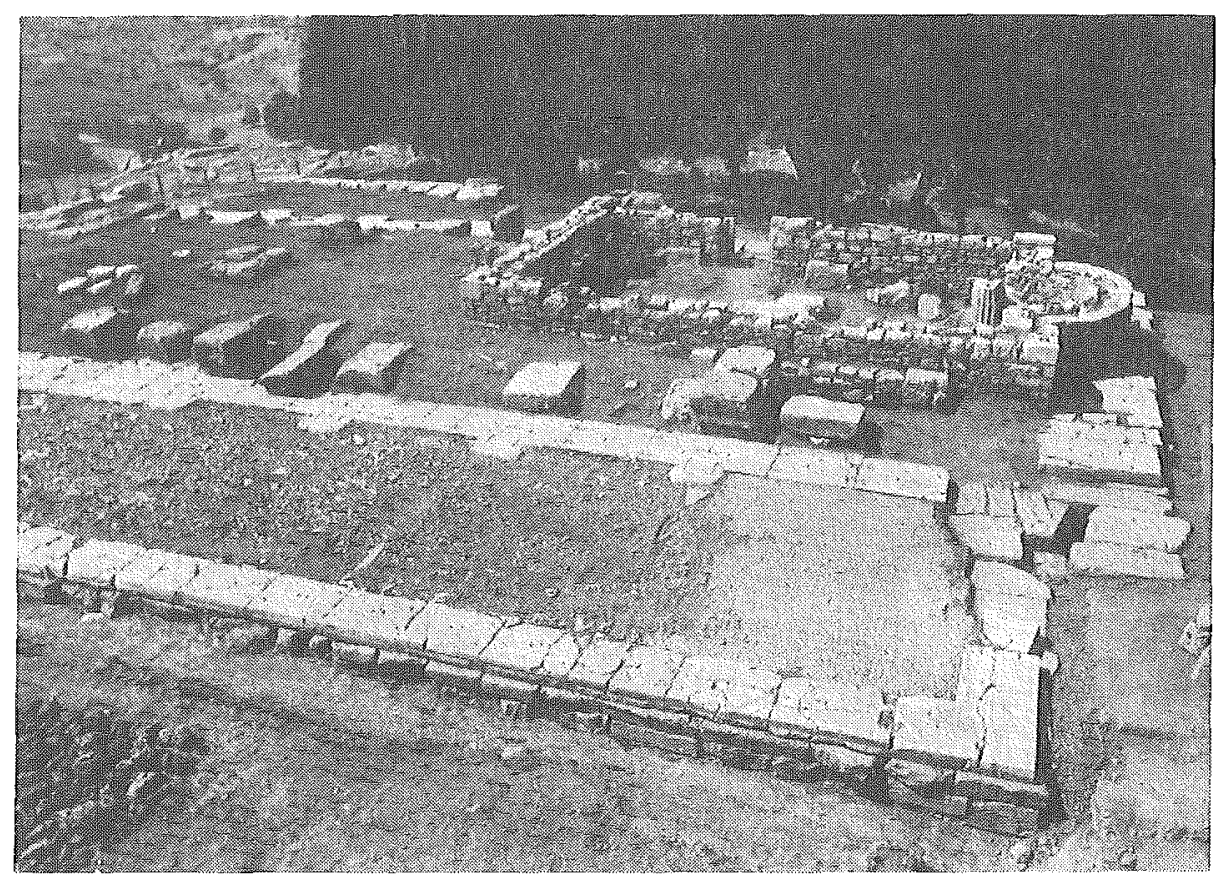

Fig. 1 : vue du temple du S.E.

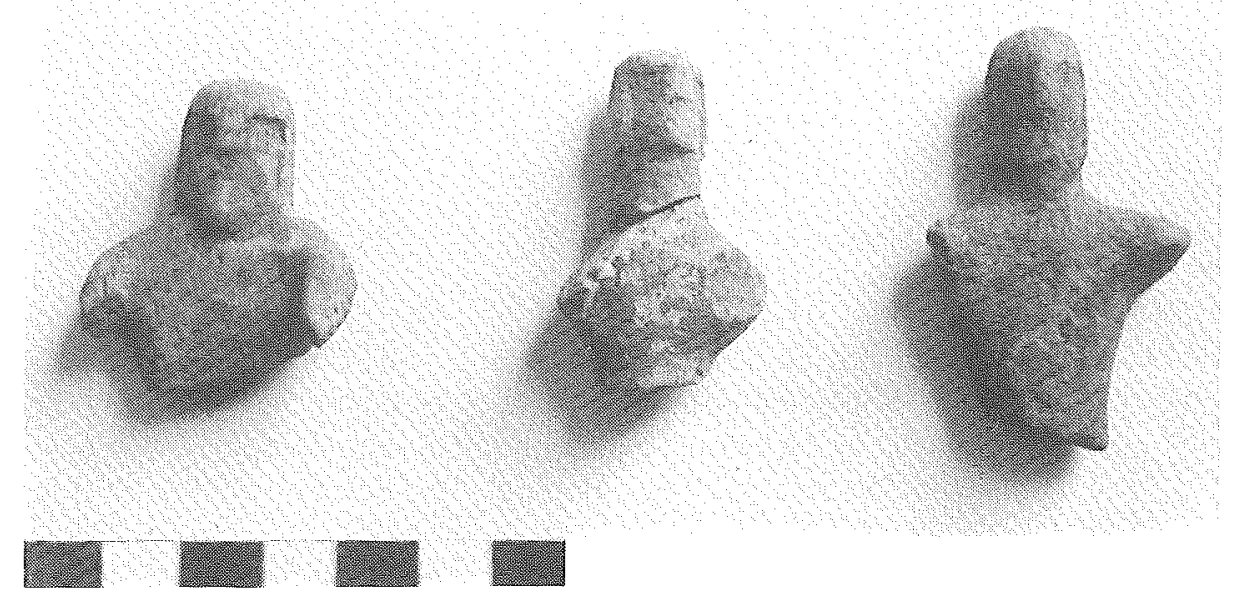

Fig. 2 : statuettes en terre-cuite portant la coiffure page (TK 5, 29, 32/1987) 

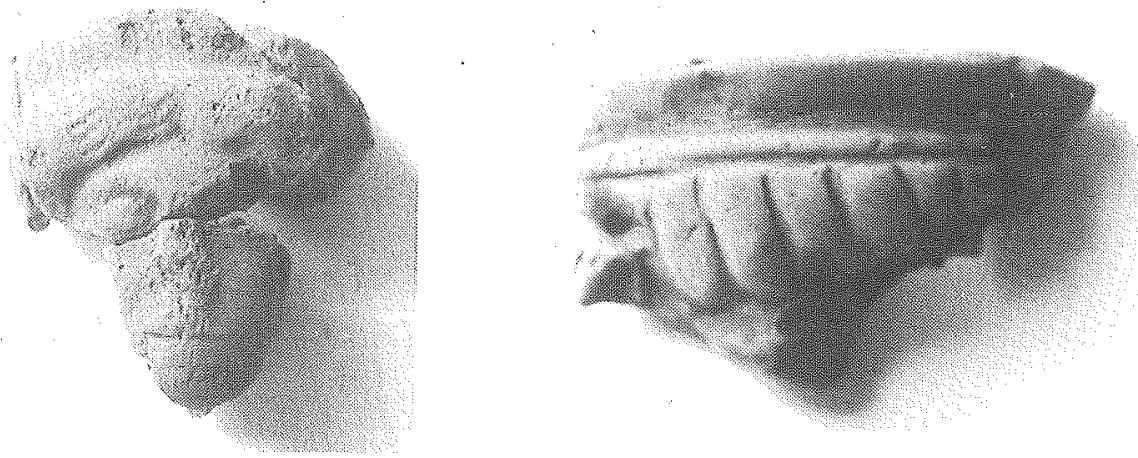

Fig. 3 : protomé féminine

Fig. 4 : fragment de protomé féminine

(TK 36/1987)

(TK 18/1987)
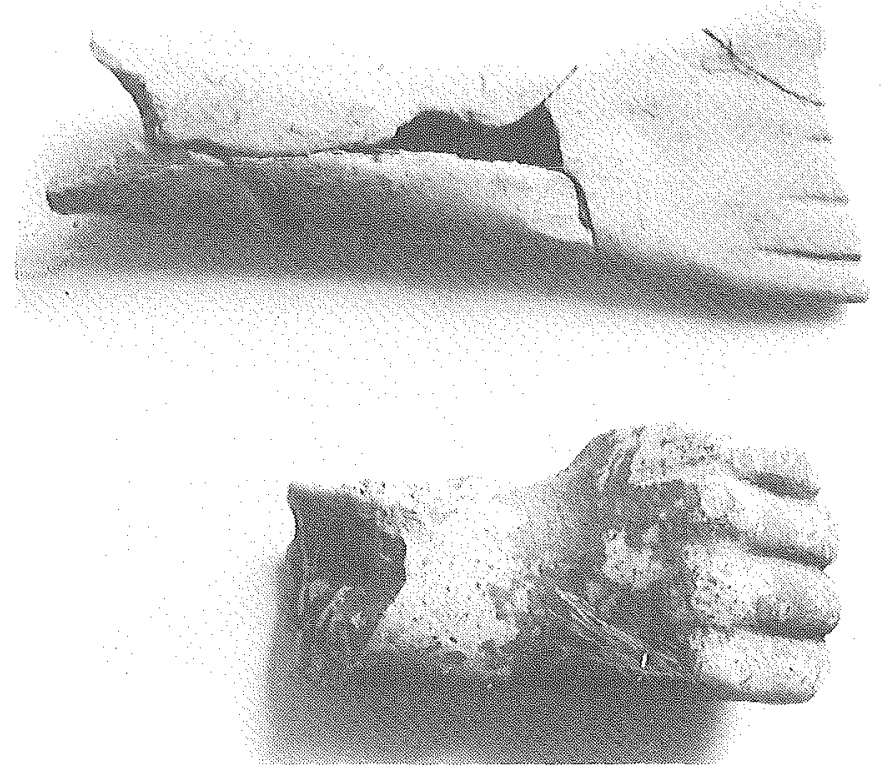

Fig. 5 : fragments d'avant-bras de statues en terre cuite (TK 30/1987 et 31a/1987)

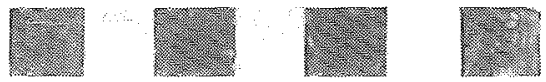



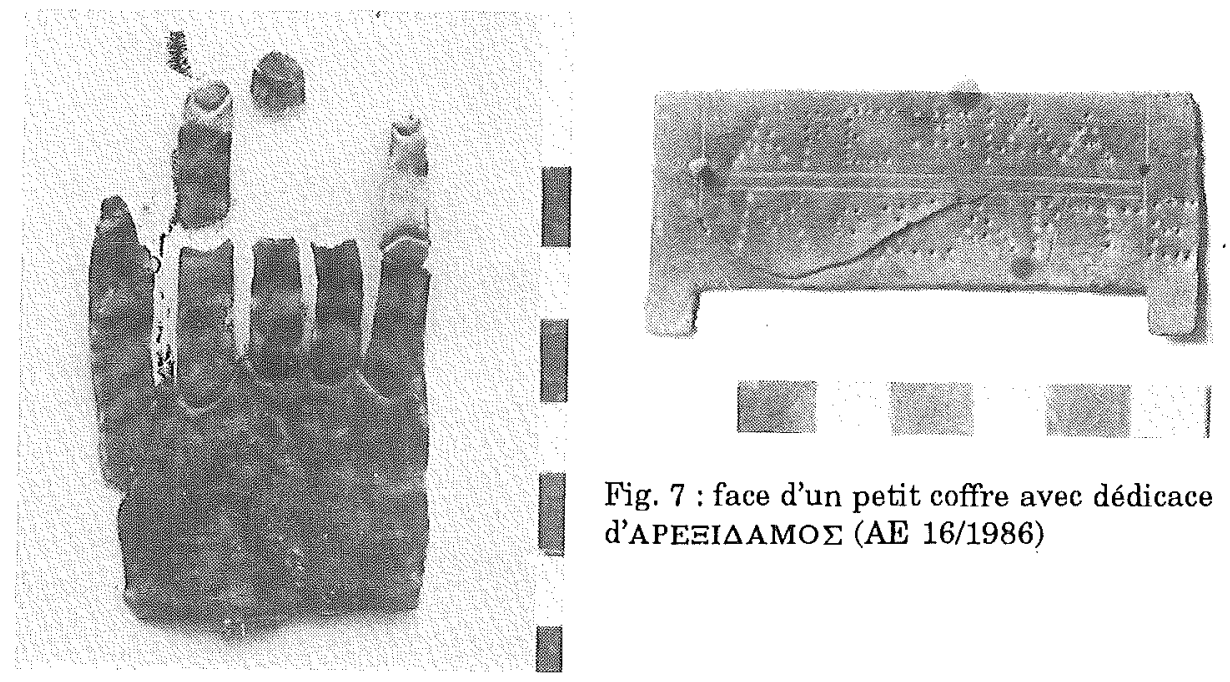

Fig. 7 : face d'un petit coffre avec dédicace d'APEEI $\triangle A M O \Sigma$ (AE 16/1986)

Fig. 6 : main en feuille de bronze (AE 4/1989)

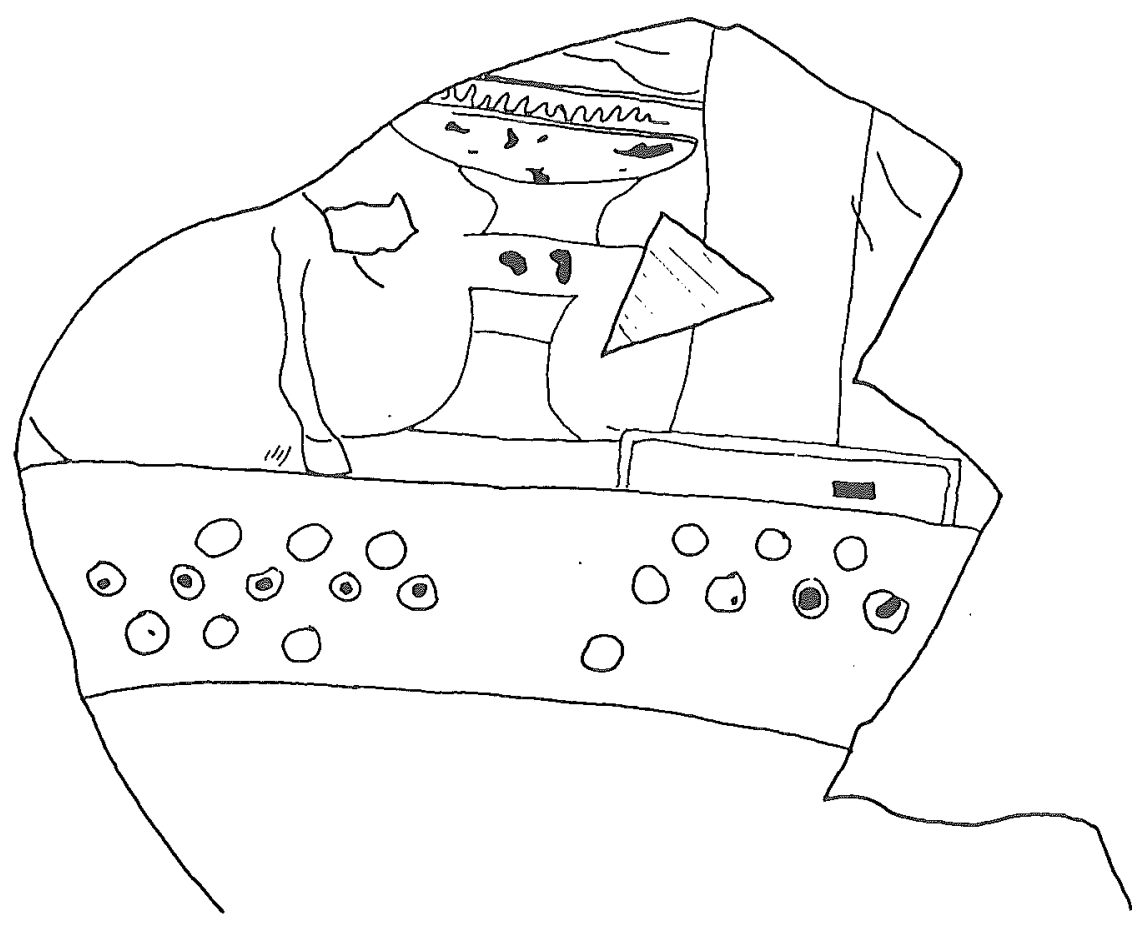

Fig. 8 : fragment d'amphore avec perirrhanterion (K 10/1987) 